Check for updates

Cite this: RSC Adv., 2017, 7, 39970

Received 30th June 2017

Accepted 1st August 2017

DOI: $10.1039 / \mathrm{c} 7 \mathrm{ra0} 07245 \mathrm{~g}$

rsc.li/rsc-advances

\section{Innovative gold-free carbon nanotube/chitosan- based competitive immunosensor for determination of HIV-related p24 capsid protein in serum}

\begin{abstract}
M. Giannetto, (D) * M. Costantini, ${ }^{*}$ M. Mattarozzi (D) and M. Careri (D)
In the past decade, the need for simple, rapid, sensitive, specific and inexpensive screening methods for diagnosis of HIV infection has led to a focus on the HIV1-related capsid protein p24. In this work, the first competitive electrochemical immunosensor for the detection of p24 in untreated human serum was developed as a simple, easy-to-use and promising tool for serum screening for early diagnosis of HIV infection. The immunodevice was implemented on disposable gold-free single-walled carbon nanotubefunctionalized screen-printed electrodes. The competitive sensor is based on the immobilization of the target protein on the electrode surface using a chitosan/glutaraldehyde crosslinking system, able to ensure, under mild conditions, a robust immobilization and a proper exposition of p24 for interaction with a mouse anti-p24 lgG1. The immunosensor setup as well as the assay's experimental conditions were then optimized, achieving a wide linear detection range of $10 \mathrm{pM}$ to $1 \mathrm{nM}$, with a low detection limit of $2 \mathrm{pM}$ in human serum. The good performance, also in terms of selectivity, trueness and precision, coupled with the advantages of an easy preparation compared to other methods requiring very complex conjugation procedures between bioreceptors and expensive nanostructures, makes the immunosensor a powerful diagnostic tool, valuable for implementation of large-scale screening programs for early diagnosis of seropositivity.
\end{abstract}

\section{Introduction}

The Human Immunodeficiency Virus (HIV) is responsible for the Acquired Immuno Deficiency Syndrome (AIDS), considered a pandemic disease by the World Health Organization (WHO). ${ }^{1,2}$

HIV occurs in two serotypes, HIV-1 and HIV-2, the first of which is the most common and more virulent and infective with respect to HIV-2. ${ }^{3}$ Globally, it is the cause of the majority of HIV infections and it is particularly common in Europe, America and central Africa.

To date, there is no drug or vaccine to treat or stop the onset of disease ${ }^{4,5}$ so prevention is the primary strategy to control HIV diffusion. An early diagnosis of infection is fundamental to prevent HIV transmission, in addition the properly timed beginning of the antiretroviral therapy allows the significant improvement of the quality and the expectancy of life for seropositive patients.

In such a context, the methods based on the research of viral nucleic acids, known as Nucleic Acid Amplification Test (NAAT), aimed at detecting virial RNA or DNA, are quite useful, ${ }^{6,7}$ allowing

Dipartimento di Scienze Chimiche, della Vita e della Sostenibilità Ambientale, Università degli Studi di Parma, Parco Area delle Scienze 17/A, 43124, Parma, Italy. E-mail: marco.giannetto@unipr.it; monica.costantini@studenti.unipr.it detection of HIV during its initial incubation stage through amplification of very low concentrations of viral nucleic acid. However, although NAATs are very sensitive, this approach is laborious and requires highly specialized technicians and expensive equipment, thus limiting its applicability for out-of-hospital testing and point-of-care service providers, especially when screening programs are implemented in resource-limited regions. ${ }^{8}$

In the past decade, the need for simple, rapid, sensitive, specific, and inexpensive screening methods for diagnosis of HIV infection has led to focus the attention on the HIV1-related capsid proteins $\mathrm{p} 24,{ }^{9}$ which are expressed in the core of the viral cell. In fact, these proteins represent useful biomarkers for early diagnosis purposes because they enter in blood-circulation since the first few weeks after infection. Nowadays, p24 proteins are widely used to develop assays having sensitivity comparable to NAATs, but with the advantage of an easy-to-use instrumentation, suitable for use in routine analysis, especially in low-resource settings with high HIV seroprevalence rates.

For this purpose, in the last years, several immunosensors aimed to the detection of p24 antigen were developed, ${ }^{10-12}$ as alternative to the already available ELISA (Enzyme Linked ImmunoSorbent Assay) kit.

Among these methods, amperometric immunosensors are highly versatile tools for rapid and user-friendly screening 
analysis of biomarkers of clinical concern, ${ }^{13}$ thanks to their compatibility with portable and compact instrumentation, exploiting disposable screen-printed electrodes as sensing substrates. ${ }^{\mathbf{1 4 , 1 5}}$ These devices match the fundamental needs required for screening analysis of biological samples in terms of reliability, simplicity of both equipment and operation, fast analysis time and cost-effectiveness, associated to good sensitivity and high specificity assured by targeted antibodies.

Concerning the immunochemical sensing devices for p24 determination, literature data mostly report about "sandwich" immunosensors; according to this approach, p24 detection is realized through the interaction with a capture antibody, immobilized on the sensing device, capable of recognizing the antigen in sample, and a secondary antibody directly tagged with a proper signalling tag, or in combination with a third tagged reading antibody.

Zheng et $a .^{\mathbf{1 6}}$ realized a sandwich HIV p24 amperometric immunosensor, implemented on gold nanoparticles-coated glassy carbon electrodes, obtaining a linear response from

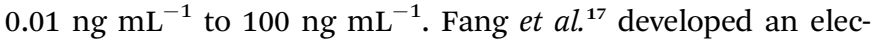
trochemical immunosensor for $\mathrm{p} 24$ detection based on graphene oxide and thionine encapsulated in carbon nanotubes-silica,

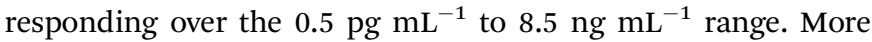
recently, Zhou and co-workers ${ }^{18}$ developed an electrochemiluminescence immunosensor for the analysis of p24, using $\mathrm{Ru}(\mathrm{bpy})_{3}{ }^{2+}$-doped silica nanoparticles and gold-nanoparticledecorated graphene, showing a linear range from $10^{-9}$ to $10^{-5} \mathrm{mg} \mathrm{mL}^{-1}$.

In this regard, it has to be noted that in all the above-cited sensors assessment of their performances was not performed in serum as real matrix, since the declared response ranges were obtained in buffered saline standard solutions. Conversely, in the present study according to the Eurachem guidelines ${ }^{19}$ and also to guidelines for bioanalytical method validation ${ }^{20}$ we devised and validated in undiluted human serum the first competitive amperometric immunosensor for determination of p24 at picomolar levels. In fact, to the best of our knowledge, there is no published research addressing the development of competitive approaches performed with amperometric immunosensors for the immunochemical determination of $\mathrm{p} 24$. The competitive assay allows the same performances of the sandwich format, but with the key advantage of requiring only one antibody, resulting in lower cost, higher simplicity and lower time involved both in the sensor setup and in the execution of the electrochemical immunoassay. Indeed, the competitive approach, based on the use of a single anti-p24 specific antibody, is undoubtedly time- and cost-saving, with respect to sandwich protocols involving the use of two complementary anti-24 antibodies able to work in combination to form the immunocomplex. Moreover, the immobilization of the "capture" antibody on the electrodic substrate, very often requires the previous chemisorption of $F_{\mathrm{c}}$-specific proteins (protein A or protein G, for example) in order to assure the proper orientation of the antibody, in terms of availability of the $F_{\mathrm{ab}}$ region for the binding of the target antigen.

In this work, the p24 antigen was immobilized on gold-free Single-Walled Carbon Nanotubes-functionalized Screen-Printed
Electrodes (SWCNT-SPEs), to take advantage of carbon nanotubes (CNT) properties, in terms of enhancement of electrochemical active area, more efficient immobilization of bioreceptors and improvement of the electronic transfer process aimed to a signal enhancement.

The immobilization of the antigen was performed using chitosan as active polymeric substrate in combination to glutaraldehyde system as cross-linker agent in order to reach under mild conditions a robust immobilization and a good exposition of p24 for the interaction with a single mouse monoclonal anti-p24 antibody. The experimental protocols involved in the realization of the immunosensor and in the execution of the assay are very simple and reproducible, avoiding complex nanostructured architectures which would require a critical control of the conjugation of antibodies and enzymes with nanomaterials.

Finally, the functionalization of the device with the target antigen, here exploited for competitive approach could be also suited for determination of anti-p24 antibodies on the same sensing substrate by easy-to-perform sample incubation, without immunocompetition.

\section{Materials and methods}

\subsection{Materials}

Human serum from clotted human male whole blood, sterilefiltered (mycoplasma tested, virus tested), Trizma ${ }^{\circledR}$ base, Tween-20, chitosan (CS, medium molecular weight), glutaraldehyde (GA, 25\% in aqueous solution), $\alpha$-Casein from bovine milk and acetic acid (99-100\% puriss.) were purchased from Sigma-Aldrich (Milan, Italy).

Recombinant (E. coli) mouse monoclonal anti-HIV-1 p24 IgG1 [39/5.4A] (anti-p24 Ab) and alkaline phosphataseconjugated rabbit anti-mouse IgG (RAM-AP) were purchased from abcam ${ }^{\circledR}$ (Cambridge, UK).

Sodium phosphate bibasic, potassium phosphate monobasic and magnesium chloride were purchased from Carlo Erba (Milan, Italy). Hydroquinone diphosphate (HQDP) and DropSens ${ }^{\circledR}$ Single-Walled Carbon Nanotubes modified ScreenPrinted Electrodes (SWCNT-SPCEs) were purchased from Metrohm Italiana (Origgio, VA, Italy).

Deionized water was obtained from an in-house Milli-Q water purification system Alpha Q-Water (Millipore, Billerica, MA, USA).

Phosphate Buffered Saline (PBS $10 \times$ ) was prepared according to the following composition: $1.37 \mathrm{M} \mathrm{NaCl}, 0.027 \mathrm{M} \mathrm{KCl}$, $0.012 \mathrm{M} \mathrm{KH}_{2} \mathrm{PO}_{4}, 0.08 \mathrm{M} \mathrm{Na}_{2} \mathrm{HPO}_{4}$ (pH 7.4). Diluted Phosphate Buffered Saline (PBS $1 \times$ ) was prepared by dilution of PBS $10 \times$ in water. The washing buffer PBS-T consisted of PBS containing $0.05 \%(\mathrm{v} / \mathrm{v})$ of the surfactant Tween-20. TRIS buffer was prepared according to the following composition: $0.1 \mathrm{M}$ Trizma ${ }^{\circledR}$ base, $0.02 \mathrm{M} \mathrm{MgCl}_{2}$ (pH 7.4). The washing buffer TRIS$\mathrm{T}$ consisted of TRIS containing $0.05 \%(\mathrm{v} / \mathrm{v})$ of the surfactant Tween-20. "Reading buffer" (RB) has the same composition of TRIS buffer but pH 9.8.

The commercial p24 ELISA Kit used for trueness assessment was purchased from abcam® (ab218268). 


\subsection{Instruments}

The immunosensor was realized using DropSens ${ }^{\circledR}$ disposable Screen-Printed Carbon Electrodes (SPCEs) modified with carboxyl-functionalized Single-Walled Carbon Nanotubes (SWCNT), assembled on ceramic substrate $(L 33 \times W 10 \times H 0.5$ $\mathrm{mm}$ ), with $4 \mathrm{~mm}$-diameter working electrode, carbon counter electrode and silver reference electrode.

All electrochemical measurements were performed with a $\mu$ Autolab III electrochemical workstation (EcoChemie, Utrecht, NL) equipped with GPES 4.0 version customized software.

\subsection{Immunosensor set-up}

SWCNT-SPCEs were coated with $15 \mu \mathrm{L}$ of $0.05 \mathrm{mg} \mathrm{mL} \mathrm{mL}^{-1} \mathrm{CS}$ dissolved in acetic acid $(0.1 \mathrm{M})$ and dried at room temperature (RT); then $30 \mu \mathrm{L}$ of $2.5 \%$ GA $(0.05 \mathrm{M})$ in PBS $1 \times$ were casted on the CS-SWCNT-SPCEs to activate the amine functions of CS, followed by washing steps $(3 \times)$ with deionized water.

Thirty $\mu \mathrm{L}$ of HIV1 p24 full length protein solution $(10 \mu \mathrm{g} \mathrm{mL}$ in PBS $1 \times$ ) were casted on the working electrode, leaved to react at RT for $1 \mathrm{~h}$ and then kept at $+4{ }^{\circ} \mathrm{C}$ overnight. After removal of unreacted p24, by accurate washing with PBS-T ( $3 \times)$ and PBS $(3 \times)$, a blocking treatment was carried out with $30 \mu \mathrm{L}$ of a $20 \mathrm{mg} \mathrm{mL}^{-1}$ solution of $\alpha$-Casein dissolved in PBS-T on each electrode for $1 \mathrm{~h}$ at RT in order to prevent a specific binding phenomena.

Then, the p24-modified SWCNT-SPCEs were used to perform the competitive electrochemical immunoassay. For this purpose, standard solutions of p24 $(30 \mu \mathrm{L})$ were mixed with $3 \mu \mathrm{L}$ of an anti-p24 Ab solution in TRIS $1 \times$ to reach a final antibody concentration of $3 \mu \mathrm{g} \mathrm{mL}{ }^{-1}$, optimized as discussed in Section 3.2. The mixture was quickly transferred on the working electrode of the immunosensor and the competition reaction was allowed to take place for $1 \mathrm{~h}$ under thermostatic conditions at $25{ }^{\circ} \mathrm{C}$. According to the competitive assay principle, as the amount of p24 in solution increases, the amount of Ab anti-p24 bound to the modified immunosensor surface decreases, being the interaction with p24 in solution favoured with respect to the binding to the immobilized antigen.

After immunocompetition, sensors were carefully washed with TRIS-T $(3 \times)$ and TRIS $(3 \times)$, to remove unreacted material. In order to detect the anti-p24 Ab immunosorbed on the electrode surface, each immunosensor was incubated for $1 \mathrm{~h}$ at $25{ }^{\circ} \mathrm{C}$ with $30 \mu \mathrm{L}$ of the enzyme-conjugated secondary reading antibody (RAM-AP), diluted in TRIS buffer by a dilution factor of 1 : 250. Finally, washing steps with TRIS-T $(3 \times)$ and TRIS $(3 \times)$ were performed to remove unreacted enzyme.

The electrochemical measurements were performed by Differential Pulse Voltammetry (DPV), scanning the potential between $-0.5 \mathrm{~V}$ and $+0.3 \mathrm{~V}$, with a pulse amplitude of $0.05 \mathrm{~V}$, a step potential of $0.005 \mathrm{~V}$ and a pulse time of $0.1 \mathrm{~s}$. Nonelectroactive HQDP was used as AP enzymatic substrate. A $1 \mathrm{mg}$ of HQDP enzyme substrate was dissolved in $1 \mathrm{~mL}$ of $\mathrm{RB}$ and drop-casted on the sensor. An equilibration time of $90 \mathrm{~s}$ and a preconditioning stage of $30 \mathrm{~s}$ at $-0.5 \mathrm{~V}$ were applied prior to run DPV in order to allow an exhaustive enzymatic reaction and to pre-concentrate HQ in its reduced form, respectively. Fig. 1

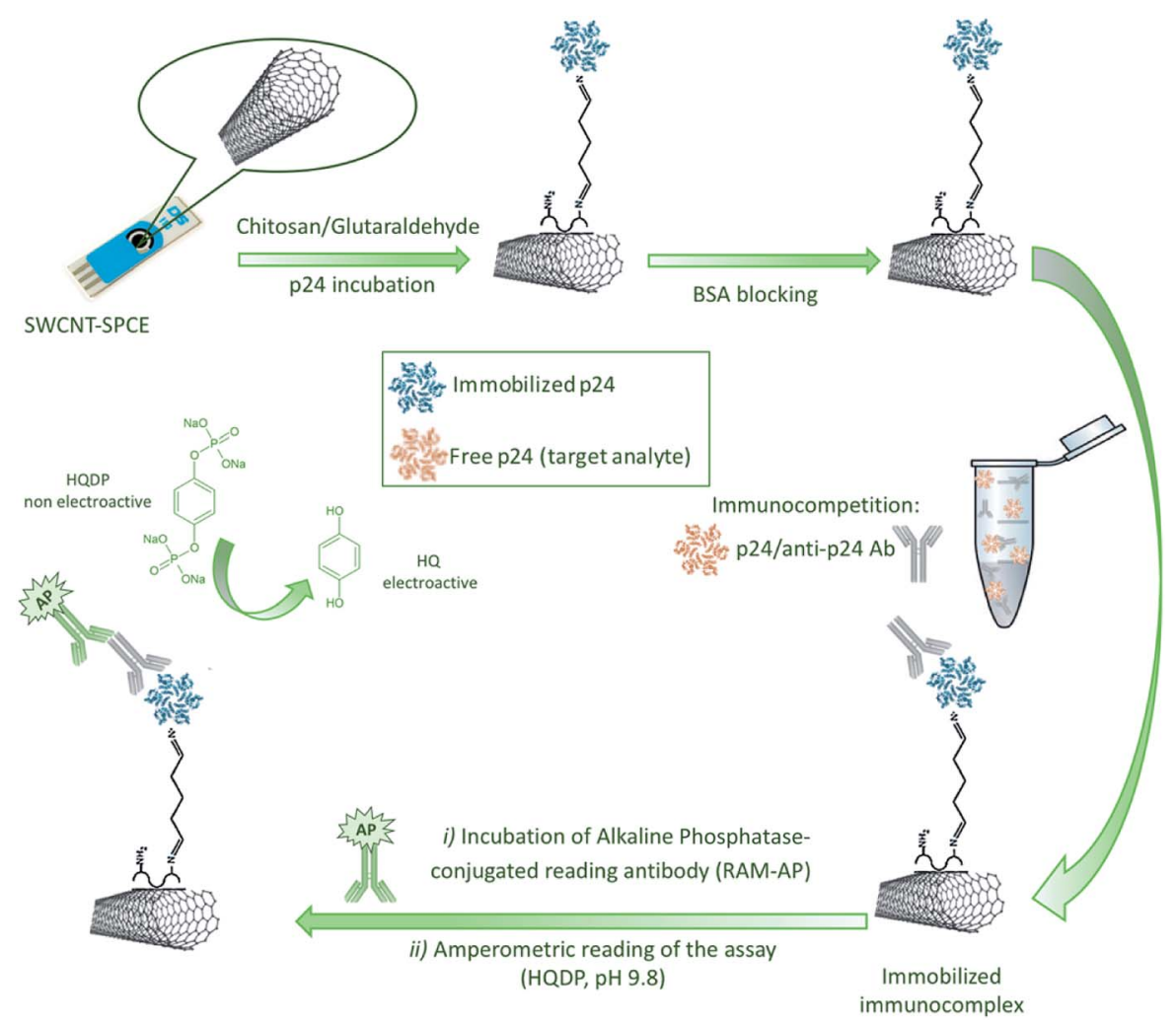

Fig. 1 Schematic representation of the realization protocol of the competitive immunosensor and set-up of the immunoassay implemented on disposable single-walled carbon nanotubes screen-printed carbon electrodes. 
shows a schematic representation of the protocol developed for the realization of the immunosensor.

The voltammetric responses were blank-normalized by dividing the current values observed for each concentration $(S)$ by the signal from blanks $\left(S_{0}\right)$, obtained only with anti-p24 without antigen in competition. The normalized signals, expressed as percentage values $\left(S / S_{0} \times 100\right)$, were plotted versus the logarithm of the p24 concentration and fitted using a four-parameter logistic function (1), as conventional for competitive immunoassays. ${ }^{21,22}$

$$
S / S_{0}=S_{\min }+\frac{\left(S_{\max }-S_{\min }\right)}{1+\left([C] / I_{50}\right)^{B}}
$$

$S_{\min }$ and $S_{\max }$ are the asymptotic minimum and maximum, respectively ( $S_{\max }$ is recorded in the absence of analyte) and $B$ is the curve slope at the inflection point $I_{50}$, corresponding to the p24 concentration $[C]$ giving a $50 \%$ of signal inhibition. Data fitting was performed by means of Microcalc OriginPro 8.5 software product (OriginLab Corporation, Northampton, MA, USA).

\subsection{Immunosensor optimization and validation}

A two-factors and 3-levels experimental design was performed to optimize the concentration of the solutions used for the immobilization of p24 on the CS/GA-modified SWCNT-SPCEs and the anti-p24 Ab in competition. Data were processed by 2way analysis of variance (ANOVA) with interactions. ANOVA was carried out using the Statgraphics Centurion XV statistical software (Statpoint Technologies Inc., Warrenton, VA, USA).

Validation of sensing device was performed according to the Eurachem guidelines ${ }^{19}$ on p24-spiked human serum, which was used as blank matrix. The detection (LOD) and quantitation (LOQ) limits were assessed as the concentration of analyte giving a signal that is $\left(3 s_{\mathrm{b}}\right) / \sqrt{ } n$ and $\left(10 s_{\mathrm{b}}\right) / \sqrt{ } n$ above the mean blank signal, respectively, where $s_{\mathrm{b}}$ is the standard deviation of the blank signal obtained from ten independent blank measurements and $n$ is the number of replicate measurements for each concentration level explored in the inhibition curve. Three replicate measurements, i.e. independent immunoassays carried out with different p24-modified CS/GA-SWCNT-SPCEs on the same specimen, were carried out for all standards and samples. Linear dynamic range was assessed as the concentration interval over which the slope of the response (i.e. parameter $B$ of the eqn (1)) is maintained within maximum deviation of $\pm 10 \%$.

Concerning stability of p24-modified CS/GA-SWCNT-SPCEs, we evaluated the shelf-life of ready-to-use sensors up to 4 weeks after blocking treatment with casein and storing them at $4{ }^{\circ} \mathrm{C}$ in the dark. After this treatment, the competitive immunoassays were carried out at regular intervals of 7 days, evaluating the inhibition rate at $I_{50}$ concentration level.

\section{Results and discussion}

\subsection{Immobilization of p 24 antigen on gold-free SWCNT- SPCES}

The most critical step in the immunosensor development was the functionalization of the electrode surface with the bio- receptor (p24 antigen) and its integration in the transduction system. Specifically, in order to allow the antigen-antibody interaction, it was fundamental to maintain the reactivity of the immobilized receptor in terms of preservation of the structural and conformational features, preventing denaturing processes that could change its immunoreactivity.

As known, the immobilization methodologies are depending on the physical and chemical characteristics of the transducer element and on the operating environmental conditions. The most used methods of functionalization are chemisorption, gel entrapment, covalent bonding and cross-linking. ${ }^{23,24}$ Concerning the last approach, a cross-linking method through chitosan and glutaraldehyde on gold-free carbon electrode substrates has been recently investigated. ${ }^{17,25}$

CS is a linear polysaccharide composed of randomly distributed $\beta$ - $(1,4)$-linked D-glucosamine and $N$-acetyl-D-glucosamine; its peculiar feature is the presence of a primary amino group at C-2 position of the glucose-amine residues, which can be suited to bind the receptor. Specifically, CS is chemisorbed on carbon substrates, whereas GA is used as cross-linker for covalent binding of bio-receptors. ${ }^{26,27} \mathrm{GA}$, being a dialdehyde, acts as bridging molecule, connecting the chitosan backbone with the bio-receptor, using the two terminal carbonyl groups, giving raise to imide bonds.

This immobilization method allowed us to efficiently functionalize the electrode surface through the organization of an ordered p24 layer that promotes a good exposure of the antigen epitopes for reaction with the target antibody; the presence of the two imide bonds and a relatively short hydrocarbon chain ensured the proper conformational freedom of the receptor, preventing folding phenomena.

In order to confirm the effective immobilization of p24 and to ensure its immunoreactivity preservation through CS/GA system, immobilized p24 versus anti-p24 "titrations" were carried out.

A first set of experiments was performed fixing the concentration of the p24 solution used for functionalization of the CS/ GA-SWCNTs-SPCEs at $0.38 \mu \mathrm{M}$ and incubating the so obtained electrodes firstly with solutions of anti-p24 Ab ranging from 0 to $117 \mu \mathrm{g} \mathrm{mL} \mathrm{m}^{-1}$ and subsequently with RAM-AP secondary antibody.

Analogous experiments were performed varying the concentration of the p24 solution used for functionalization of the CS/GA-SWCNT-SPCEs from 0 to $3.85 \mu \mathrm{M}$, keeping constant the concentration of anti-p24 at $10 \mu \mathrm{g} \mathrm{mL}{ }^{-1}$. In both cases, the obtained DPV signals increased versus increasing anti-p24 Ab and p24 concentrations. In addition, negligible signals were obtained in the absence of both anti-p24 Ab and p24, evidencing the proper immobilization of the bioreceptor on the electrode surface and the fact that non-specific binding does not occur.

\subsection{Optimization of the immunocompetition parameters}

The experimental conditions related to the performance of the developed immunosensor were optimized in serum matrix with the final goal to reach a dynamic response range suitable for diagnostic purposes. 
The experimental design was aimed at finding the optimal conditions to maximize the signal inhibition rate, associated to a fixed concentration of the antigen in competition. For this purpose, the optimal concentrations of both the immobilized p24 and the anti-p24 antibody were assessed by means of a twofactors and 3-levels experimental design procedure. Immunocompetition experiments were carried out keeping the concentration of p24 in competition constant at 500 pM and varying the concentrations of the solution used for p24 immobilization (immobilized p24) and anti-p24 Ab added to the sample. The experimental domain explored for the optimization is described in Table 1. Three replicated measurements were carried out with independent sensors for each experimental condition. The dataset from the experimental design was processed by means of 2-ways ANOVA with interactions. The ANOVA results showed that both factors (immobilized p24 and anti-p24 Ab) as well as their interaction have a significant effect $(p<0.05)$ on the immunosensor response (Fig. 2). On the basis of these results, p24 at $0.38 \mu \mathrm{M}$ and anti-p24 $\mathrm{Ab}$ at $3 \mu \mathrm{g} \mathrm{mL}{ }^{-1}$ were found as the optimal concentrations for the immunocompetition. Moreover, even though under these conditions the best inhibition rate values were observed for both medium (0) and low (-) concentrations of $\mathrm{p} 24$, the concentration of $0.38 \mu \mathrm{M}$ (medium level) was definitively chosen as optimal, due to the better signal to noise ratio.

\subsection{Immunosensor performance in human serum}

In order to assess the immunoreactivity preservation of the antigen-antibody interaction in untreated human serum samples, a preliminary titration experiment was carried out on CS/GA-SWCNT-SPCEs modified with p24: for this purpose, different concentrations of the anti-p24 antibody ranging from 0 to $5 \mu \mathrm{g} \mathrm{mL}{ }^{-1}$ were tested. As reported in Fig. 3, no significant matrix effect was found to affect the immunosensor response, as evidenced by the negligible signal obtained in the absence of anti-p24 antibody; serum matrix did not influence the antigenantibody interaction, as confirmed by the dynamic response observed under concentrations changes of anti-p24 Ab.

On the basis of these findings, different immunocompetition assays were carried out on serum samples spiked with standard p24 over the $20 \mathrm{fM}$ to $35 \mathrm{nM}$ concentration range. Since p24 present in the sample was found to inhibit antibody

Table 1 Three levels of the two factors optimized in the experimental design; $(+)=$ high level, $(0)=$ medium level, $(-)=$ low level

\begin{tabular}{lll}
\hline Experiment number & $\begin{array}{l}\text { Immobilized p24 } \\
\text { concentration }(\mu \mathrm{M})\end{array}$ & $\begin{array}{l}\text { Anti-p24 concentration } \\
\left(\mu \mathrm{g} \mathrm{mL}^{-1}\right)\end{array}$ \\
\hline 1 & $3.85(+)$ & $117(+)$ \\
2 & $3.85(+)$ & $30(0)$ \\
3 & $3.85(+)$ & $3(-)$ \\
4 & $0.38(0)$ & $117(+)$ \\
5 & $0.38(0)$ & $30(0)$ \\
6 & $0.38(0)$ & $3(-)$ \\
7 & $0.038(-)$ & $117(+)$ \\
8 & $0.038(-)$ & $30(0)$ \\
9 & $0.038(-)$ & $3(-)$
\end{tabular}

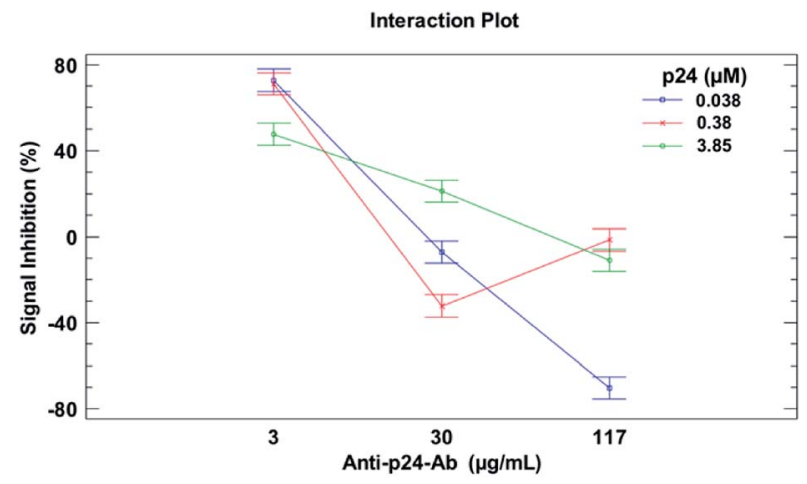

Fig. 2 Interaction plot from 2-way ANOVA, evidencing the effects of anti-p24 Ab and immobilized p24 antigen concentrations on the signal inhibition extent.

binding to the immobilized p24, increasing analyte concentration resulted to reduce the amount of anti-p24 bound to the modified surface of the sensor, as shown by the DPV scans (Fig. 4).

Voltammetric data were interpolated according to eqn (1) giving the inhibition curve reported in Fig. 5.

The immunosensor showed very good analytical performance in terms of sensitivity, linear dynamic range, precision and trueness. In addition, as previously observed, in the present work, differently from previously reported electrochemical sensors for p24 determination, ${ }^{15-17}$ validation was carried out in serum matrix in such a way to be useful as quality criteria in routine analysis. Wide linear detection range from $10 \mathrm{pM}$ to $1 \mathrm{nM}$ with LOD of $2 \mathrm{pM}$ and LOQ of $26 \mathrm{pM}$ was obtained when analyzing p24-spiked human serum, an excellent result if compared to the previous developed electrochemical sensors based on sandwich approach involving complex conjugation procedures between nanostructures and biomolecules and validated only in matrix-free standard buffered aqueous solutions. A sensitivity of $0.82 \pm 0.12 S / S_{0} \times \mathrm{M}^{-1}$ was assessed from the parameter B obtained in the data fitting shown Fig. 5.

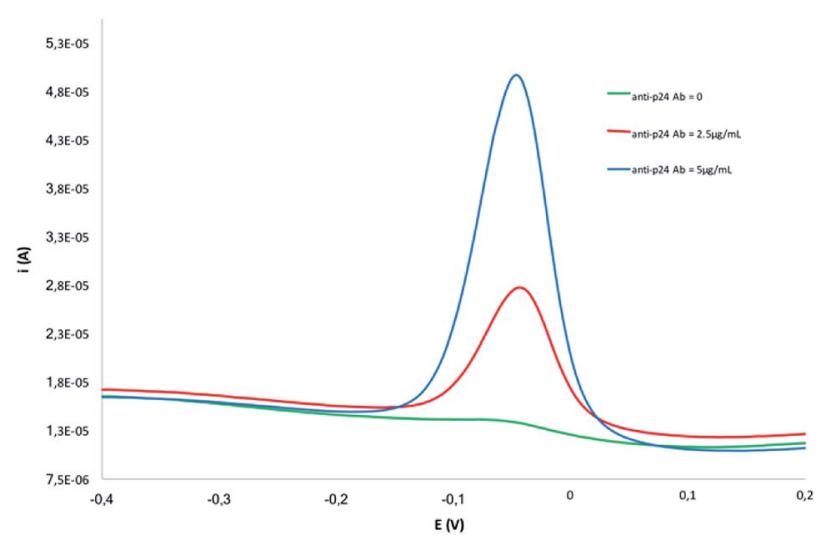

Fig. 3 DPV scans recorded in human serum over titration with different concentration of anti-p24 Ab carried out on CS/GA-SWCNTSPCEs usually modified with p24. 


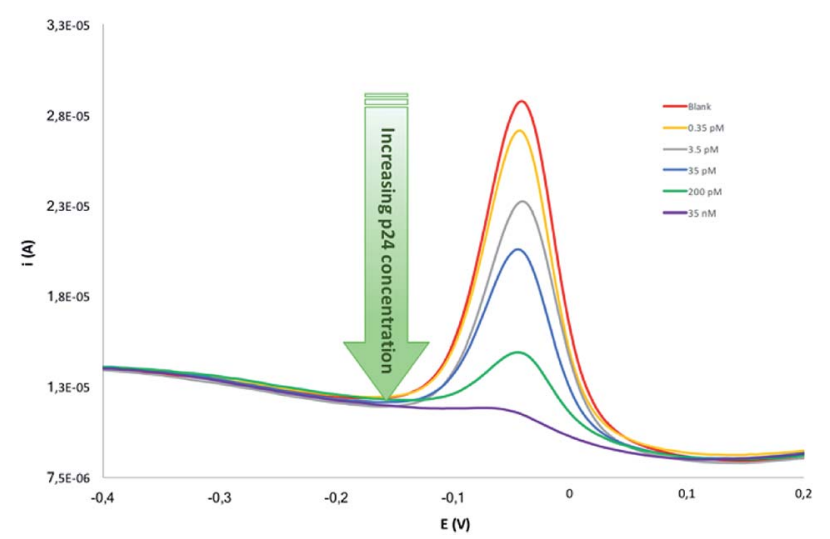

Fig. 4 Selection of DPV scans recorded carrying out immunocompetition assays on serum samples, spiked with standard p24 over the $20 \mathrm{fM}$ to $35 \mathrm{nM}$ concentration range.

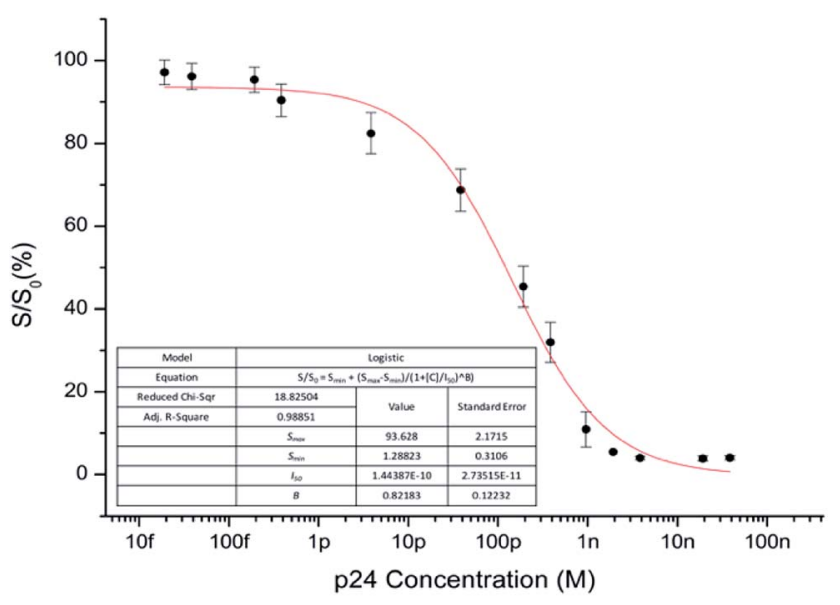

Fig. 5 Inhibition curve for $\mathrm{p}-24$ spiked undiluted human serum, obtained interpolating the immunocompetition responses over the $20 \mathrm{fM}$ to $35 \mathrm{nM}$ concentration range, using the four-parameter logistic function (1). Inset table: fitted curve parameters.

Concerning the intermediate precision calculated on three independent immunosensing assays, relative standard deviations (RSD) always lower than $5 \%$ were observed over the explored concentration range.

According to Eurachem guidelines, ${ }^{19}$ trueness was assessed by spiking human serum with p 24 standard at concentration levels not included in the calibration dataset in order to cover the dynamic response range, i.e. close to LOQ, $I_{50}$ and $S_{\min }$ values. The fortified samples were analysed through immunocompetition on p24-modified CS/GA-SWCNT-SPCEs and the inhibition rates were interpolated according to the equation included in Fig. 5, giving the recovery rates reported in Table 2. Recoveries were between $89 \pm 3 \%$ and $112 \pm 5 \%$, confirming that the proposed competitive immunosensor is applicable to real samples, thus giving accurate quantitative results in undiluted human serum.

The same fortified samples were also analysed through a commercial ELISA kit from abcam ${ }^{\circledR}$ (ab218268) based on a sandwich protocol for p24 determination in serum and other
Table 2 Trueness of the competitive immunosensor assessed by analysis of p24-spiked human serum

\begin{tabular}{lcc}
$\begin{array}{l}\text { Spiked p24 } \\
\text { (in human serum) }\end{array}$ & Found p24 & \\
\hline $50 \mathrm{pM}$ & $56 \pm 2.3 \mathrm{pM}$ & \% Recovery rate \\
$150 \mathrm{pM}$ & $161 \pm 5.62 \mathrm{pM}$ & $112 \pm 5$ \\
$1 \mathrm{nM}$ & $0.89 \pm 0.03 \mathrm{nM}$ & $89 \pm 3$
\end{tabular}

${ }^{a}$ Analysed with p24-modified CS/GA-SWCNT SPCEs and interpolated according to the inhibition curve reported in Fig. $5 .{ }^{b}$ Recovery rates (\%) calculated as $(100 \times$ found value/spiked value $)$.

biological fluids. The samples were processed according to the protocol endorsed from the manufacturer, giving results below the LOQ of the kit for the first two spiked levels (50 and $150 \mathrm{pM}$ ), while the $1 \mathrm{nM}$-spiked serum sample gave an estimated concentration of $0.97 \pm 0.11 \mathrm{nM}$, in good agreement with the result obtained with our immunosensor $(0.89 \pm 0.03 \mathrm{nM}$, as reported in Table 2). These findings confirm the trueness of the developed competitive immunosensor, also when compared to the results obtained with an external reference method, based on the use of quite different protocols and antibodies.

\subsection{Immunosensor selectivity}

Immunosensor selectivity was assessed evaluating the signals obtained from proteins markers, which can potentially occur in the samples to be analyzed, as Bovine Serum Albumin (BSA), carcinoembryonic antigen (CEA) and Carbohydrate Antigen 125 (CA125). For this purpose, the immunocompetition experiments were carried out with each interferent at $I_{50}$ concentration levels. In each case, no significant inhibition of signal $(p>0.05)$ with respect to blanks containing only anti-p24 was obtained. Furthermore, mixing p24 at $I_{50}$ level with the potentially interferent markers at the same concentration, an inhibition rate of about $50 \%$ was observed for all the mixtures. All of these results confirm the good selectivity, as well as the absence of a specific response of the competitive immunosensor.

\subsection{Immunosensor stability}

To evaluate immunosensor stability, the p24-modified CS/GASWCNT-SPCEs electrodes blocked with $\alpha$-Casein were stored at $4{ }^{\circ} \mathrm{C}$, in the dark, for different days $(7,14,21$ and 28 days) and then used to perform the immunocompetitive measurements. Specifically, focusing the attention on the experiments carried out over four weeks, inhibition rates not significantly different $(p>0.05)$ from $50 \%$ were obtained at $I_{50}$ concentration level after 7, 14, 21 and 28 days (with independent sensors). These findings prove the good stability and the activity retention of the developed immunosensor up to one month of storage.

\section{Conclusions}

The first competitive immunosensor for determination of HIVrelated p24 capsid protein foreshadows as a promising diagnostic tool for detection of such an infection biomarker at 
subnanomolar levels in human serum, showing high sensitivity, selectivity and robustness.

From the comparison with previously reported sandwich immunosensors for p24 determination, involving very expensive and complex conjugations between bioreceptors and nanostructured substrates, the present competitive immunosensors offers comparable if not even better analytical performance assessed for the first time by validation in untreated human serum matrix.

The competitive device could be also proposed for the determination of anti-p24 antibodies on the same sensing substrate by direct serum incubation in the absence of immunocompetition, making the immunosensor a very versatile diagnostic tool for widespread application for biomedical purposes.

Finally, the performance of the realized device, coupled with the advantages of an easy preparation, low-cost and portability of the instrumentation, makes it an analytically robust diagnostic tool valuable for implementation of large-scale screening programs for early diagnosis of seropositivity.

\section{Conflicts of interest}

There are no conflicts to declare.

\section{Notes and references}

1 E. A. Emini, The Human Immunodeficiency Virus: Biology, Immunology, and Therapy, Princeton University Press., Princeton, 2002.

2 L. Montagnier, Science, 2002, 298, 1727-1728.

3 J. A. Levy, Aids, 1993, 7, 1401-1410.

4 A. J. McMichael, Annu. Rev. Immunol., 2006, 24, 227-255.

5 D. A. Garber, G. Silvestri and M. B. Feinberg, Lancet Infect. Dis., 2004, 4, 397-413.

6 D. Daskalakis, Top. Antivir. Med., 2010, 19, 18-22.

7 S. M. Owen, Curr. Opin. HIV AIDS, 2012, 7, 125-130.

8 M. P. Busch, Dev. Biol., 2007, 127, 87-112.

9 K. Teeparuksapun, M. Hedstrom, E. Y. Wong, S. Tang, I. K. Hewlett and B. Mattiasson, Anal. Chem., 2010, 82, 8406-8411.
10 F. Kheiri, R. E. Sabzi, E. Jannatdoust, E. Shojaeefar and H. Sedghi, Biosens. Bioelectron., 2011, 26, 4457-4463.

11 N. Gan, X. Du, Y. Cao, F. Hu, T. Li and Q. Jiang, Materials, 2013, 6, 1255-1269.

12 Y. Zhang, H. Yang, J. Yu and H. Wei, Anal. Bioanal. Chem., 2016, 408, 6115-6121.

13 M. Giannetto, M. Mattarozzi, E. Umiltá, A. Manfredi, S. Quaglia and M. Careri, Biosens. Bioelectron., 2014, 62, 325-330.

14 R. A. S. Couto, J. L. F. C. Lima and M. B. Quinaz, Talanta, 2016, 146, 801-814.

15 O. D. Renedo, M. A. Alonso-Lomillo and M. J. Martínez, Talanta, 2007, 73, 202-219.

16 L. Zheng, L. Jia, B. Li, B. Situ, Q. Liu, Q. Wang and N. Gan, Molecules, 2012, 17, 5988-6000.

17 Y. S. Fang, X. J. Huang, L. S. Wang and J. F. Wang, Biosens. Bioelectron., 2015, 64, 324-332.

18 L. Zhou, J. Huang, B. Yu, Y. Liu and T. You, ACS Appl. Mater. Interfaces, 2015, 7, 24438-24445.

19 Eurachem Guide, The Fitness for Purpose of Analytical Methods - A Laboratory Guide to Method Validation and Related Topics, 2nd edn, 2014, http://www.eurachem.org.

20 Guidance for Industry: Bioanalytical Method Validation, Food and Drug Administration, revision 1, 2013, available from, https:/www.fda.gov/downloads/Drugs/Guidances/ ucm368107.pdf.

21 C. March, J. J. Manclús, Y. Jiménez, A. Arnau and A. Montoya, Talanta, 2009, 78, 827-833.

22 A. Manfredi, M. Giannetto, M. Mattarozzi, M. Costantini, C. Mucchino and M. Careri, Anal. Bioanal. Chem., 2016, 408, 1-10.

23 W. H. Scouten, J. H. Luong and R. S. Brown, Trends Biotechnol., 1995, 13, 178-185.

24 S. K. Sharma, N. Sehgal and A. Kumar, Curr. Appl. Phys., 2003, 3, 307-316.

25 Y. Ma, X. L. Shen, Q. Zeng, H. S. Wang and L. S. Wang, Talanta, 2017, 164, 121-127.

26 O. A. Monteiro and C. Airoldi, Int. J. Biol. Macromol., 1999, 26, 119-128.

27 Y. Liu, Y. Li, S. Liu, J. Li and S. Yao, Biomaterials, 2004, 25, 5725-5733. 